\title{
Surgery time interval and molecular subtype may influence Ki67 change after core needle biopsy in breast cancer patients
}

Xiaosong Chen ${ }^{1 *}$, Siji Zhu' ${ }^{1}$ Xiaochun Fei ${ }^{2}$, David H. Garfield ${ }^{3}$, Jiayi Wu' ${ }^{1}$ Ou Huang ${ }^{1}$, Yafen Li ${ }^{1}$, Li Zhu', Jianrong $\mathrm{He}^{1}$, Weiguo Chen ${ }^{1}$, Xiaolong $\mathrm{Jin}^{2}$ and Kunwei Shen ${ }^{1^{*}}$

\begin{abstract}
Background: To investigate the accuracy of core needle biopsy (CNB) in evaluating breast cancer estrogen receptor (ER), progesterone receptor (PR), HER2, and Ki67 status and to identify factors which might be associated with Ki67 value change after CNB.

Methods: A retrospective study was carried out on 276 patients with paired CNB and surgically removed samples (SRS). Clinico-pathological factors as well as the surgery time interval (STI) between CNB and surgery were analyzed to determine whether there were factors associated with Ki67 value change after CNB. Five tumor subtypes were classified as follows: Luminal A, Luminal B-HER2-, Luminal B-HER2+, Triple Negative (TN), and HER2+. Ki67 value change was calculated as SRS minus CNB.

Results: Mean STI after CNB was 4.5 (1-37) days. Good agreement was achieved for ER, PR, and HER2 evaluation between CNB and SRS. However, Ki67 expression level was significantly higher in SRS compared with CNB samples: $29.1 \%$ vs. $26.2 \%(P<0.001)$. Both univariate and multivariate analysis demonstrated that STI and molecular subtype were associated with a Ki67 change after CNB. Luminal A tumors experienced more Ki67 elevation than Luminal B-HER2- diseases $(6.2 \%$ vs $-0.1 \%, P=0.014)$. Patients with longer STI after CNB had a higher Ki67 increase: $-1.1 \%$ within $1-2$ days, $2.1 \%$ with 3-4 days, and $5.6 \%$ more than 4 days, respectively $(P=0.007)$. For TN and HER2+ tumors, the Ki67 change was apt to be 0 with STI $\leq 4$ days, while a $>7 \%$ Ki67 increase was noticed in patients with $\mathrm{STI} \geq 5$ days.
\end{abstract}

Conclusion: CNB was accurate in evaluating ER, PR, HER2, and molecular subtype status. Ki67 value significantly increased after CNB, which was associated with STI and molecular subtype. Further translational research needs to consider Ki67 changes following CNB among different breast cancer molecular subtypes.

Keywords: Breast cancer, Core needle biopsy, Ki67 change, Molecular subtype, Surgery time interval

\footnotetext{
*Correspondence: chenxiaosong0156@hotmail.com; kwshen@medmail.com.cn

'Comprehensive Breast Health Center, Ruijin Hospital, Shanghai Jiaotong

University School of Medicine, 197 Ruijin Er Road, Shanghai 20025, China

Full list of author information is available at the end of the article
} 


\section{Background}

Core needle biopsy (CNB) is recommended for an initial breast cancer pathological diagnosis and is used to evaluate estrogen receptor (ER), progesterone receptor (PR), and HER2 status [1]. Microarray data have identified that breast cancer is comprised of at least five molecular subtypes: Luminal A, Luminal B, triple negative (TN), HER2 positive and normal-like [2]. The 2013 St. Gallen breast cancer consensus recommends using ER, PR, HER2, and Ki67 results to classify breast cancer into molecular subtypes in order to guide systemic treatment decision making [3]. Our previous study showed that CNB had a high concordance rate in evaluating molecular subtype status compared with those in surgically removal samples (SRS) [4].

With the development of new agents, "window of opportunity" pre-surgical trials have been applied to test their potential anticancer abilities and mechanisms in breast cancer patients [5]. Patients in these trials are usually treated with experimental agents for a relatively short period compared with standard neoadjuvant systemic therapy [6]. In this situation, response rate is no longer suitable as an endpoint, so a breast cancer proliferation biomarker, such as Ki67, is then applied to determine the new agent's biologic effect [7]. Thus, a Ki67 change after two weeks of endocrine treatment, for example, may predict response rates in a neoadjuvant study [8]. In addition, a decrease of Ki67 after neoadjuvant chemotherapy or endocrine therapy seems also to be related to a good prognosis $[9,10]$.

However, tumor heterogeneity, sample fixation, and CNB methods can cause discordance of biomarkers evaluation between CNB and SRS [4]. Also, Ki67, compared with ER, PR, and HER2, is reported to have only a fair to moderate agreement between CNB and SRS, especially in ER+/Luminal breast cancers [11, 12]. Furthermore, several studies have demonstrated that Ki67 expression will increase after $\mathrm{CNB}$, which may be caused by biopsy stimulation, arguing that this Ki67 change needs to be considered in clinical practice as well as in "window of opportunity" trials $[11,13]$. However, there are limited data about which factors are associated with Ki67 change after CNB. Therefore, we performed a comprehensive analysis to find which factor(s) can influence Ki67 change after CNB in early breast cancer patients.

\section{Methods}

\section{Patient population}

Consecutive breast cancer patients who received CNB and followed by surgery in Ruijin Hospital, Shanghai Jiaotong University School of Medicine between Oct. 2009 and Feb. 2012 were retrospectively analyzed. All enrolled patients needed paired CNB and SRS samples. Patients with large tumor were likely to receive CNB by surgeon's choice. Ultrasound was applied to guide the $\mathrm{CNB}$ procedure, with more than three 14-gauce CNB samples being collected for pathological examination. CNB and SRS samples were fixed in $10 \%$ neutral buffered formalin within $30 \mathrm{~min}$ after tumor removal, and fixation intervals ranged from at least $6 \mathrm{~h}$ to $24 \mathrm{~h}$ for $\mathrm{CNB}$ and at least $6 \mathrm{~h}$ to $48 \mathrm{~h}$ for SRS samples. Patients' enrollment criteria were described in our previous report [11]. In addition, CNB and surgery dates were retrieved to calculate the surgery time interval (STI) after CNB. Twenty-two patients with STI more than 60 days were further excluded. All participants gave written informed consent before inclusion. The independent Ethical Committee/Institutional Review Board of Ruijin Hospital, Shanghai Jiaotong University School of Medicine reviewed and approved this study protocol, which was conducted in accordance with the Declaration of Helsinki.

\section{Breast cancer molecular subtype classification}

The methods and positivity criteria for immunohistochemical (IHC) assessment of ER, PR, HER2, and Ki67 were described in our previous report, all of which were performed in the Department of Pathology, Ruijin Hospital, Shanghai Jiaotong University School of Medicine [11]. In brief, Ventana Autostain System (BenchMark XT, Ventana Medical Systems, Inc., Tucson, AZ) was used to stain the paired CNB and SRS, which were further evaluated by two senior pathologists (X. Fei, and X. Jin). Tumors with more than $1 \%$ positive invasive cell nuclear staining were classified as ER+ or PR+. The 2007 ASCO/ CAP (American Society of Clinical Oncology/College of American Pathologists) guidelines were applied in the HER2 status evaluation. Either HER2 IHC 3+ or fluorescence in situ hybridization positivity was regarded as HER2 positive (HER2+) [14]. For Ki67 expression scoring, we used the same method for calculating CNB and SRS samples. Cell distribution over the entire slice was first reviewed and 500-2000 cells were chosen from different microscopic views if the Ki67 expression distribution was uniform. Otherwise, 2000 cells were equally counted in both hotspot and negative areas in slice. Ki67 expression was scored as the percentage of positive invasive tumor cells with any nuclear staining and recorded as mean percentage of positive cells [11]. Histo-pathological parameters and receptor status in CNB were set as the baseline. Ki67 change between CNB and SRS was calculated by using $\mathrm{CNB}$ as the baseline.

Hormonal receptor negativity (HR-) was defined as both ER- and PR-. The concordance rate for molecular subtype classification between CNB and SRS was similar by using a Ki67 value of either $14 \%$ or $20 \%$, while the latter had the higher $\mathrm{K}$ value [11]. Also, $20 \%$ was the mean value for $H R+/ H E R 2$ - patients and the median value for all patients in CNB samples. Thus, $20 \%$ was 
selected as the Ki67 cutoff value in determining Luminal status. Five breast cancer molecular subtypes were classified according to the 2013 St. Gallen breast cancer consensus [3]: Luminal A (ER+/HER2-, Ki67 $<20 \%$ and $\mathrm{PR} \geq 20 \%$ ), Luminal B-HER2- (ER+/HER2-, Ki67 $\geq 20 \%$ or ER+/HER2-, PR $<20 \%$, or ER-/PR+/HER2-), Luminal B-HER2+ (HR+/HER2+), TN (HR-/HER2-) and HER2+ (HR-/HER2+).

\section{Statistical analysis}

Kappa test was applied to test concordance rates for ER, PR, HER2, and molecular subtypes between CNB and SRS. Values of $\kappa>0.6$ were correlated with good agreement, values between 0.4 and 0.6 considered moderate agreements, values $<0.4$ corresponded to fair, and values $<0.2$ reflected poor agreement. Ki67 change after CNB was compared by using two paired samples $t$ test. Chi-square test was used to calculate the association between STI and tumor characteristics. ANOVA analysis was performed to calculate the relationship between Ki67 change and potential influencing factors including: age, menopausal status, surgery type, histopathology, tumor grade, tumor size, lymph node status, ER, PR, HER2, molecular subtype, and STI. Multivariate ANOVA analysis was then done to find the association and interaction between Ki67 change and these factors. The SPSS statistical software package (version 13.0; SPSS Company, Chicago, IL) was used in the statistical analysis and two-sided $P$ values less than 0.05 regarded as statistically significant.

\section{Results}

\section{Patient characteristics}

A total of 276 breast cancer patients were enrolled. Mean age was 56.6 (24-91) years. Ninety percent of patients were diagnosed with invasive ductal carcinoma and $32.6 \%$ had grade III tumors. There were $214(77.5 \%)$ and 163 (59.1 \%) of cases with ER and PR positive disease. Fiftynine (21.4 \%) had HER2+ breast cancer. Mean Ki67 value was $26.2 \%$ (1-90 \%) in CNB samples, and $53.3 \%$ tumors were classified as Ki67 high expression. There were 73 (26.4\%), 109 (39.5\%), 33 (12.0\%), 35 (12.7\%), and 26 (9.4\%) patients classified as Luminal A, Luminal B-HER2-, Luminal B-HER2+, TN, and HER2+ subtype, respectively. Mean STI after CNB was 4.5 (1-37) days. Ten patients had STI of more than 10 days. The first, second, and third quartile days of STI were 3,4 , and 5 days, respectively. Next, we categorized STI as following groups: less than 3 days (55 patients), 3-4 days (113 patients), more than 4 days (108 patients) (Table 1 ). Table 2 shows STI categorized versus initial tumor characteristics as well as patient characteristics. There was no association between STI groups and clinic-pathological characteristics. Regarding Ki67 expression level at CNB and at surgery versus patients' time to surgery, there was no significant correlation between Ki67 expression level and STI (Fig. 1a and b).

\section{Comparison of receptor status and $\mathrm{Ki} 67$ results between CNB samples and SRS}

Concordance rates of ER, PR, and HER2 between CNB and SRS were $94.2 \%, 87.0 \%$ and $97.1 \%$. Kappa test showed $\mathrm{K}$ values were $0.841,0.729$, and 0.914 , respectively, demonstrating good overall agreement. Additionally, good agreement was observed for HR test, with a concordance rate of $94.2 \%(\kappa=0.837)$. In terms of molecular subtype analysis, the overall concordance rate was $72.5 \%$, with $\mathrm{k}$ value of 0.630 , also regarded as a good agreement.

Ki67 expression value was much higher in SRS compared with CNB samples by using two paired samples $t$ test, with mean values of $29.1 \%$ and $26.2 \%$, respectively $(\mathrm{P}<0.001)$. Using $20 \%$ as the cutoff value for a high level of Ki67 expression, the concordance rate was $80.4 \%$, with $\mathrm{k}$ value of 0.60 . TN breast cancer had the highest Ki67 value of all subtypes. Median and mean Ki67 change was 0 (inter-quartile range (IQR), $-4.5 \%$, $10 \%)$ and $2.9 \%( \pm 13.2 \%)$, respectively.

\section{Factors associated with Ki67 change analysis}

Univariate ANOVA analysis was used to determine whether patient characteristics and STI were associated with Ki67 changes. Both breast cancer molecular subtype and STI were significantly associated with Ki67 change after CNB, while other host and tumor characteristics had no influence (Figs. 2, 3, Table 3). Fig. 1c shows Ki67 change after CNB versus patients' STI. Most cases had Ki67 change between $-20 \%$ and $20 \%$ after CNB. Mean Ki67 change with different STIs after CNB was: $-1.1 \%$ (1-2 days STI), $2.1 \%$ (3-4 days STI), $5.6 \%$ ( $\geq 5$ days STI), respectively $(P=0.007$, Table 4). Subgroup analysis showed that patients receiving surgery more than 4 days after $\mathrm{CNB}$ had a higher Ki67 increase compared with those treated with surgery within 2 days $(P=0.006)$. Besides, we classified STI into another 5 groups: $1-2$ days $(n=55)$, 3 days $(n=50), 4$ days $(n=63), 5$ days $(n=52)$, and $\geq$ 6 days $(n=56)$. ANOVA analysis still showed that Ki67 change after $\mathrm{CNB}$ was significantly associated with STI $(\mathrm{P}=0.01$, Additional file 1: Figure S1). Luminal BHER2- tumors, which had a higher baseline Ki67 value than Luminal A disease, showed a Ki67 decrease after CNB. However, other breast cancer subtypes showed an increased Ki67 value, with a mean Ki67 absolute increase from $3.3 \%$ to $6.2 \%$ (Table 5). Subgroup comparison showed that Luminal A tumors had a higher Ki67 value increase after CNB than Luminal B-HER2- $(P=0.014)$. 
Table 1 Baseline patient characteristics

\begin{tabular}{|c|c|c|}
\hline Characteristic & No. & Percent \\
\hline Age, years & $56.6(24-91)$ & \\
\hline$<40$ & 20 & 7.2 \\
\hline $40-49$ & 64 & 23.2 \\
\hline $50-70$ & 155 & 56.2 \\
\hline$>70$ & 37 & 13.4 \\
\hline \multicolumn{3}{|l|}{ Menstrual status } \\
\hline Peri/pre-menopause & 89 & 32.2 \\
\hline Post-menopause & 187 & 67.8 \\
\hline \multicolumn{3}{|l|}{ Breast surgery type } \\
\hline Mastectomy (+/-reconstruction) & 239 & 86.6 \\
\hline Lumpectomy & 37 & 13.4 \\
\hline \multicolumn{3}{|l|}{ Pathological type } \\
\hline Invasive ductal carcinoma & 246 & 89.1 \\
\hline Invasive lobular carcinoma & 12 & 4.3 \\
\hline Others & 18 & 6.5 \\
\hline \multicolumn{3}{|l|}{ Tumor size } \\
\hline Tx & 5 & 1.8 \\
\hline$\leq 2 \mathrm{~cm}$ & 118 & 42.8 \\
\hline $2-5 \mathrm{~cm}$ & 147 & 53.3 \\
\hline$>5 \mathrm{~cm}$ & 6 & 2.2 \\
\hline \multicolumn{3}{|l|}{ Axillary lymph node } \\
\hline Negative & 155 & 56.2 \\
\hline Positive & 121 & 43.8 \\
\hline \multicolumn{3}{|l|}{ Histologic grading } \\
\hline । & 6 & 2.2 \\
\hline$\|$ & 134 & 48.6 \\
\hline III & 90 & 32.6 \\
\hline NA & 46 & 16.7 \\
\hline \multicolumn{3}{|l|}{ Estrogen Receptor } \\
\hline Negative & 62 & 22.5 \\
\hline Positive & 214 & 77.5 \\
\hline \multicolumn{3}{|l|}{ Progesterone Receptor } \\
\hline Negative & 113 & 40.9 \\
\hline Positive & 163 & 59.1 \\
\hline \multicolumn{3}{|l|}{ Hormonal Receptor } \\
\hline Negative & 61 & 22.1 \\
\hline Positive & 215 & 77.9 \\
\hline \multicolumn{3}{|l|}{ HER2 } \\
\hline Negative & 217 & 78.6 \\
\hline Positive & 59 & 21.4 \\
\hline Ki67 (\%, mean) & $26.2(1-90)$ & \\
\hline$<20$ & 129 & 46.7 \\
\hline$\geq 20$ & 147 & 53.3 \\
\hline
\end{tabular}

Table 1 Baseline patient characteristics (Continued)

\begin{tabular}{lll}
\hline Molecular subtype & & \\
Luminal A & 73 & 26.4 \\
Luminal B-HER2- & 109 & 39.5 \\
Luminal B-HER2+ & 33 & 12.0 \\
Triple negative & 35 & 12.7 \\
HER2 positive & 26 & 9.4 \\
Surgery time interval (days) & $4.5(1-37)$ & \\
$1-2$ & 55 & 19.9 \\
$3-4$ & 113 & 40.9 \\
$\geq 5$ & 108 & 39.1 \\
\hline
\end{tabular}

Abbreviation: NA not available

Multivariate ANOVA analysis demonstrated that STI and molecular subtype were still significantly associated with Ki67 change after CNB, with $P$ values of 0.010 and 0.042 , respectively. Subgroup test showed that there was a slight trend of interaction between STI and molecular subtype for Ki67 change $(P=0.220)$. Furthermore, we analyzed Ki67 changes among various subtypes with different STI (Fig. 4). For HER2+ or TN patients, Ki67 change was apt to be 0 among those receiving surgery within 4 days after $\mathrm{CNB}$, while the Ki67 value increase was $11.5 \%$ and $7.7 \%$, respectively, in those with a STI of more than 4 days (Fig. 4).

\section{Discussion}

Our present study demonstrated that CNB was accurate in evaluating breast cancer receptor and molecular subtype status compared with subsequent SRS. More importantly, we found that both STI and molecular subtype were associated with Ki67 changes after CNB. Luminal A tumors had more Ki67 elevation than did Luminal B-HER2- tumors. The Ki67 value increase was much more obvious in patients with prolonged STI. TN or HER2+ breast cancer patients were more likely to experience a significant Ki67 increase with a long surgery waiting time.

CNB has been proven to be a minimally invasive and accurate method in preoperative pathological diagnosis [15], and can provide sufficient tissue for breast cancer biomarkers analysis, including ER, PR, HER2, and Ki67 [1]. Meta-analysis has shown that CNB is reliable in testing ER, PR, and HER2 status compared with SRS [4]. Moreover, CNB has good agreement with SRS in breast cancer molecular subtype analysis in determining ER, PR, HER2, and Ki67 results [11]. There is no consensus concerning the Ki67 cutoff value for high proliferation [3]. In our study, we used the $20 \%$ as the cutoff value, the median value in CNB samples and which had a relatively high concordance rate for molecular subtype 
Table 2 Surgery time interval and tumor characteristics

\begin{tabular}{|c|c|c|c|c|}
\hline Characteristic & $\begin{array}{l}1-2 \\
\text { days }\end{array}$ & $\begin{array}{l}3-4 \\
\text { days }\end{array}$ & $\geq 5$ days & $P$ value \\
\hline Age & & & & 0.067 \\
\hline$<40$ & 5 & 5 & 10 & \\
\hline $40-49$ & 16 & 24 & 24 & \\
\hline $50-70$ & 32 & 70 & 53 & \\
\hline$>70$ & 2 & 14 & 21 & \\
\hline Menstrual status & & & & 0.084 \\
\hline Peri/pre-menopause & 24 & 30 & 35 & \\
\hline Post-menopause & 31 & 83 & 73 & \\
\hline Breast surgery type & & & & 0.960 \\
\hline $\begin{array}{c}\text { Mastectomy } \\
\text { (+/-reconstruction) }\end{array}$ & 47 & 98 & 94 & \\
\hline Lumpectomy & 8 & 15 & 14 & \\
\hline Pathological type & & & & 0.973 \\
\hline Invasive ductal carcinoma & 49 & 101 & 96 & \\
\hline Invasive lobular carcinoma & 3 & 5 & 4 & \\
\hline Others & 3 & 7 & 8 & \\
\hline Tumor size & & & & $0.110^{\mathrm{a}}$ \\
\hline Tx & 2 & 2 & 1 & \\
\hline$\leq 2 \mathrm{~cm}$ & 30 & 45 & 43 & \\
\hline $2-5 \mathrm{~cm}$ & 23 & 65 & 49 & \\
\hline$>5 \mathrm{~cm}$ & 0 & 1 & 5 & \\
\hline Axillary lymph node & & & & 0.582 \\
\hline Negative & 28 & 67 & 60 & \\
\hline Positive & 27 & 46 & 48 & \\
\hline Histologic grading & & & & $0.821^{a}$ \\
\hline I & 0 & 3 & 3 & \\
\hline$\|$ & 30 & 51 & 53 & \\
\hline III & 15 & 41 & 34 & \\
\hline NA & 10 & 18 & 18 & \\
\hline Estrogen Receptor & & & & 0.732 \\
\hline Negative & 11 & 28 & 23 & \\
\hline Positive & 44 & 85 & 85 & \\
\hline Progesterone Receptor & & & & 0.357 \\
\hline Negative & 21 & 52 & 40 & \\
\hline Positive & 34 & 61 & 68 & \\
\hline Hormonal Receptor & & & & 0.822 \\
\hline Negative & 11 & 27 & 23 & \\
\hline Positive & 44 & 86 & 85 & \\
\hline HER2 & & & & 0.797 \\
\hline Negative & 43 & 91 & 83 & \\
\hline Positive & 12 & 22 & 25 & \\
\hline Ki67 (\%, mean) & & & & 0.222 \\
\hline$<20$ & 20 & 55 & 54 & \\
\hline
\end{tabular}

Table 2 Surgery time interval and tumor characteristics (Continued)

\begin{tabular}{lllll}
\hline$\geq 20$ & 35 & 58 & 54 & \\
$\begin{array}{l}\text { Molecular subtype } \\
\text { Luminal A }\end{array}$ & 13 & 30 & 30 & \\
Luminal B-HER2- & 26 & 43 & 40 & \\
Luminal B-HER2+ & 5 & 13 & 15 & \\
Triple negative & 4 & 18 & 13 & \\
HER2 positive & 7 & 9 & 10 & \\
\hline
\end{tabular}

Abbreviation: NA not available

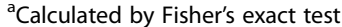

analysis between CNB and SRS. The 2013 St. Gallen breast cancer consensus of $20 \%$ Ki67 cutoff value was then applied to classify patients into various molecular subtypes. Our concordance analysis demonstrated that $\mathrm{CNB}$ was accurate in determining molecular subtype status compared with SRS.

Ki67, a well-established proliferation marker, is used to determine specific breast cancer Luminal subtypes [16]. For early breast cancer patients, high Ki67 expression is associated with a poor outcome [17]. Several retrospective studies have found that Ki67 could predict endocrine treatment and chemotherapy response in ER+ breast cancer patients $[8,18]$. In addition, Ki67 is a key proliferation marker for calculating breast cancer recurrence score in the Oncotype-DX assay [19]. However, several studies have found that there was only fair to moderate agreement for Ki67 testing between CNB and SRS, and that the $k$ value was much lower than ER, PR, and HER2 evaluation; this was mainly interpreted as sampling error or tumor heterogeneity $[11,12]$. Most studies have demonstrated that Ki67 expression will increase after CNB, possibly due to wound healing [20] or sample fixation intervals difference between $\mathrm{CNB}$ and surgically removed samples, which warrants further study. Our current data also showed a significantly higher Ki67 expression value in SRS compared with CNB samples ( $29.1 \%$ vs. $26.2 \%)$.

In preoperative "window of opportunity" clinical studies, Ki67 is often used as a surrogate biomarker to evaluate new anticancer drug anti-proliferation ability [7]. Patients treated with neoadjuvant endocrine therapy have a higher response rate if they experience a Ki67 decrease after 2 weeks therapy [8]. However, all such studies omitted Ki67 change after $\mathrm{CNB}$, which may result in a shift of efficacy analysis. We analyzed potential clinic-pathological factors associated with Ki67 change after CNB to determine if any subgroup might change significantly and found that breast cancer molecular subtype was, indeed, an independent factor. Patients with different subtypes 

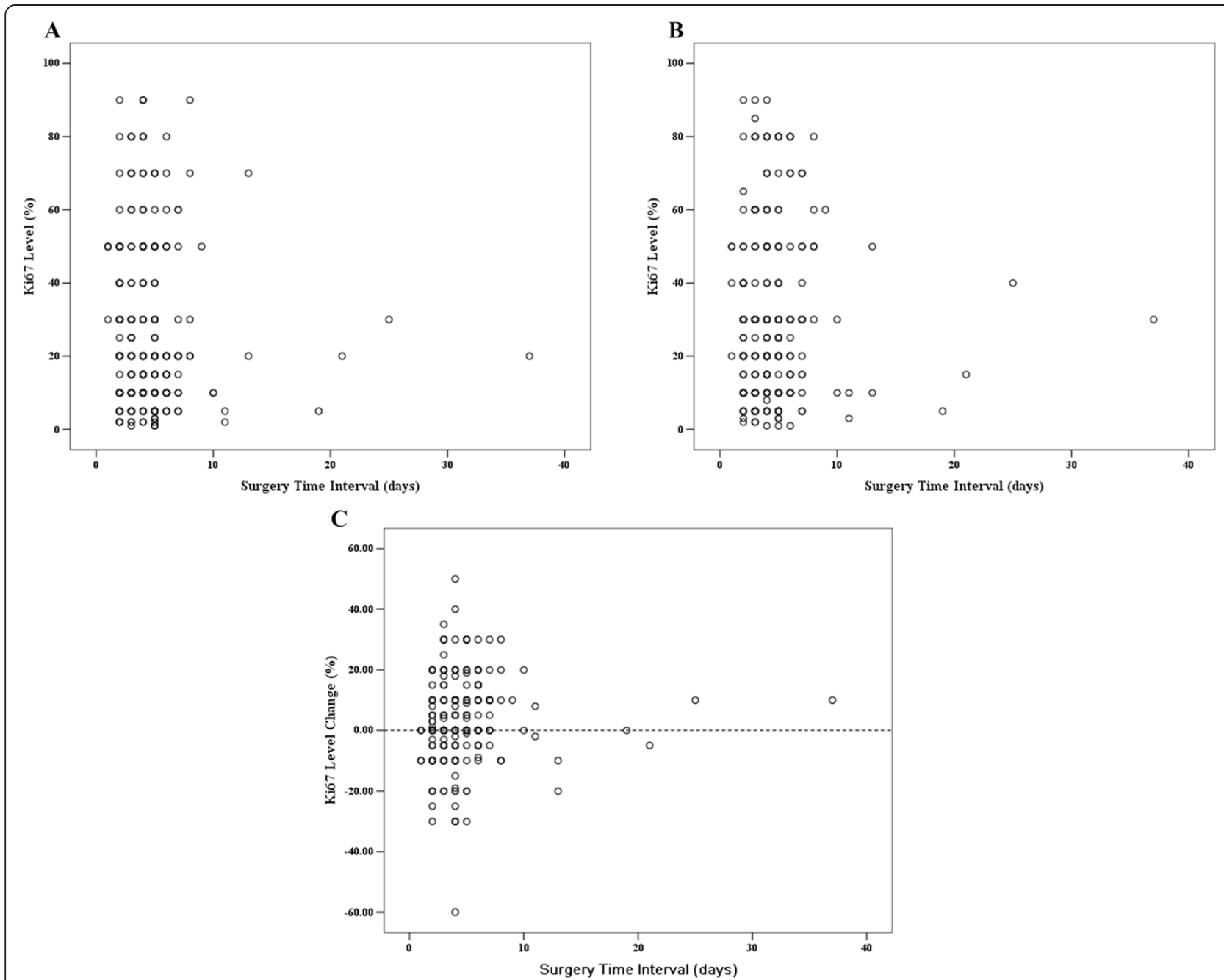

Fig. 1 Ki67 expression level and change distribution at different surgery time intervals. a): Ki67 expression level of core needle biopsies versus surgery time intervals; b): Ki67 expression level at surgically removed samples versus surgery time intervals; c): Ki67 change distribution versus surgery time intervals

did have various Ki67 changes, indicating that Ki67 change seemed to be associated with its specific tumor biologic behavior. In neoadjuvant treatment of HER2-positive breast cancer, gene expression profiling analysis has demonstrated that subtype status can significantly change after treatment. Luminal $\mathrm{B}$ or HER2-enriched tumors on CNB could become Luminal A, perhaps due to cell reprogramming, stromal alteration, or heterogeneity. Luminal A tumors on CNB had more discordant cases and changes to other subtypes in SRS [21]. Our present study showed that Luminal A tumors had a higher Ki67 increase after CNB compared with Luminal B-HER2-, perhaps also reflecting wound healing, stromal reaction, or tumor heterogeneity. This indicates that further translational research needs to interpret anti-proliferation efficacy among the different molecular subtypes.
It has been reported that Ki67 changes after CNB were more obvious in TN or HER2+ breast cancer, but that there was no significant Ki67 increase in the luminal subtypes [13]. The major difference between the two studies was STI after CNB. There were 41 days in that study, much longer than in our study, which had a mean of only 4.5 days. Although, no significant Ki67 changes were noted over time after CNB in that study, we here report that STI after CNB is significantly associated with Ki67 change. Thus, breast cancer patients with longer surgery waiting time after CNB had a higher chance of Ki67 increases. Furthermore, we analyzed whether STI had different effects on Ki67 change among the breast cancer molecular subtypes. Ki67 change was apt to be 0 in TN and HER2 breast cancer patients treated with surgery within 4 days after $\mathrm{CNB}$, while this was $\geq 7 \%$ in patients with STI more than 4 days, similar to the other 


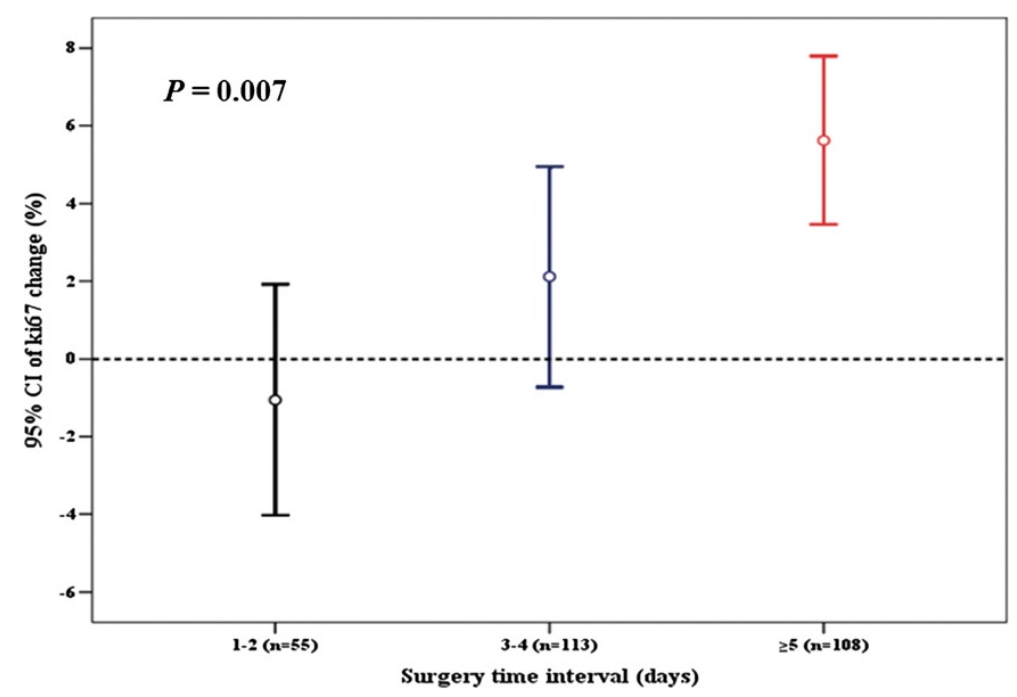

Fig. 2 Surgery time interval and Ki67 change after core needle biopsy

study. Although Ki67 increase after CNB has not been reported to cause a worse disease outcome, its increase has still been associated with chemotherapy sensitivity [10]. Moreover, a long interval between surgery and adjuvant chemotherapy has been reported to cause a worse prognosis in HER2+ and TN breast cancer patients [22]. Taken together, our data support that TN or HER2+ breast cancer patients may need to be treated with surgery within a short interval after $\mathrm{CNB}$, and that neoadjuvant systemic therapy may be a reasonable option for these patients if they must wait a long time for surgery [23].

There were several limitations in our study. First, the number of enrolled patients was not large enough to further explore subgroup analysis differences, especially for subgroup interaction effect analysis. There were relatively few patients in separate subgroups regarding various STI and molecular subtype combinations. In addition, most patients were treated with surgery with a

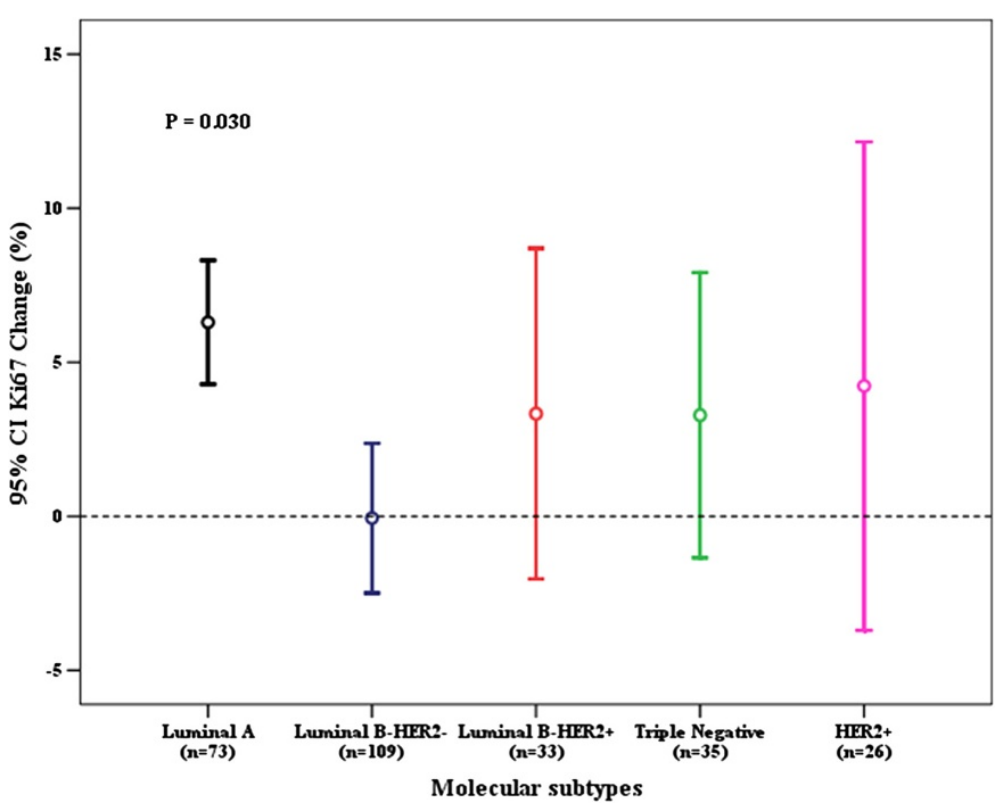

Fig. 3 Molecular subtypes and Ki67 change after core needle biopsy 
Table 3 Univariate analysis of Ki67 change and clinic-pathological factors

\begin{tabular}{lc}
\hline Clinic-pathological factors & $P$ value \\
\hline Age & 0.521 \\
Menopause status & 0.638 \\
Tumor size & 0.658 \\
Lymph node status $^{2}$ & 0.358 \\
Estrogen receptor $^{\mathrm{a}}$ & 0.529 \\
Progesterone receptor $^{\mathrm{a}}$ & 0.585 \\
HER2 $^{\mathrm{a}}$ & 0.569 \\
Molecular subtype $^{\mathrm{a}}$ & 0.030 \\
Surgery time interval & 0.007 \\
\hline
\end{tabular}

${ }^{a}$ Expression status in CNB sample

Univariate ANOVA analysis used to analyze association between Ki67 change and clinico-pathological factors

very short waiting time after $\mathrm{CNB}$, which may be different from other centers. In our center, we firstly did diagnostic hematoxylin and eosin staining for tumor within $24 \mathrm{~h}$ after biopsy in the in-patient ward, and further surgery was arranged for most patients when invasive breast cancer was found. ER, PR, and HER2 status results was not mandatory before surgery. For other centers with longer waiting periods after biopsy, in which IHC or FISH analysis was done before surgery, one would to consider the STI differences in order to

Table 4 Ki67 expression and change value at CNB and SRB in different surgery time intervals

\begin{tabular}{llll}
\hline & No. & Median Ki67 \% (IQR) & Mean Ki67 \% (SD) \\
\hline $\begin{array}{lll}\text { All populations } \\
\text { CNB }\end{array}$ & 276 & & $26.2(22.0)$ \\
SRS & & $25(10,40)$ & $29.1(22.0)$ \\
Ki67 change ${ }^{a}$ & & $0(-4.5 .10)$ & $2.9(13.2)$ \\
1-2 days & 55 & & $27.7(21.0)$ \\
CNB & & $20(10,40)$ & $26.6(19.1)$ \\
SRS & & $20(10,40)$ & $-1.1(11.0)$ \\
Ki67 change ${ }^{a}$ & & $0(-10,5)$ & $28.4(24.6)$ \\
$3-4$ days & 113 & & $30.5(23.0)$ \\
CNB & & $20(10,50)$ & $2.1(15.2)$ \\
SRS & & $25(10,50)$ & \\
Ki67 change ${ }^{a}$ & & $0(-5,10)$ & $23.2(20.8)$ \\
$\geq 5$ days & 108 & & $28.8(22.4)$ \\
CNB & & $17.5(10,30)$ & $5.6(11.4)$ \\
SRS & & $25(10,40)$ & \\
Ki67 change & & $5(0,10)$ & \\
\hline
\end{tabular}

${ }^{a}$ Ki67 change, SRS minus CNB

Abbreviation: $C N B$ core needle biopsy, IQR inter quartile range, SRS surgically removed samples, $S D$ standard deviation
Table 5 Ki67 expression and change value of CNB and SRS among molecular subtypes

\begin{tabular}{|c|c|c|c|}
\hline & No. & Median Ki67 (IQR) & Mean Ki67(SD) \\
\hline Luminal A & 73 & & \\
\hline CNB & & $10(5,10)$ & $8.2(4.1)$ \\
\hline SRS & & $10(10,20)$ & $14.5(9.6)$ \\
\hline Ki67 change ${ }^{a}$ & & $5(0,10)$ & $6.3(8.6)$ \\
\hline Luminal B HER2- & 109 & & \\
\hline CNB & & $20(10,40)$ & 26.9 (19.5) \\
\hline SRS & & $20(10,30)$ & 26.9 (19.2) \\
\hline Ki67 change ${ }^{a}$ & & $0(-10,10)$ & $-0.1(12.8)$ \\
\hline Luminal B HER2+ & 33 & & \\
\hline CNB & & $20(10,50)$ & $30.2(20.7)$ \\
\hline SRS & & $30(15,55)$ & $33.5(20.0)$ \\
\hline Ki67 change ${ }^{a}$ & & $0(-10,14.5)$ & $3.3(15.1)$ \\
\hline Triple negative & 35 & & \\
\hline CNB & & $60(30,80)$ & $54.1(26.4)$ \\
\hline SRS & & $70(30,80)$ & $57.4(25.8)$ \\
\hline Ki67 change ${ }^{a}$ & & $0(-10,10)$ & $3.3(13.5)$ \\
\hline HER2 positive & 26 & & \\
\hline CNB & & $25(20,40)$ & $31.2(17.3)$ \\
\hline SRS & & $30(23.75,50)$ & $35.4(15.1)$ \\
\hline Ki67 change ${ }^{a}$ & & $5(-1.25,20.0)$ & $4.2(19.6)$ \\
\hline
\end{tabular}

${ }^{a}$ Ki67 change, SRS minus CNB

Abbreviation: CNB core needle biopsy, IQR inter quartile range, SRS surgically removed samples, $S D$ standard deviation

compare our results to theirs. Our mean STI was 4.5 days and only 3 separate groups were constructed with different STIs. We have not further categorized patients into another group with longer STI because there were only 10 patients with STI of more than 10 days. This prevented our study from being able to answer whether Ki67 change would be decreased in specific subtypes with a prolonged surgical waiting period. Finally, HER2 status was only evaluated by the 2007 edition of ASCO/CAP criteria, with there being a slight difference with new edition of ASCO/CAP recommendation [24]. However, we doubt that this would change our results.

\section{Conclusion}

Our study has demonstrated that CNB is accurate in evaluating ER, PR, HER2, and molecular subtype status in breast cancer. Ki67 value can significantly increase after CNB, and this is associated with STI and molecular subtype. Luminal A tumors experienced more Ki67 elevation than did Luminal B-HER2-. Breast cancer patients 


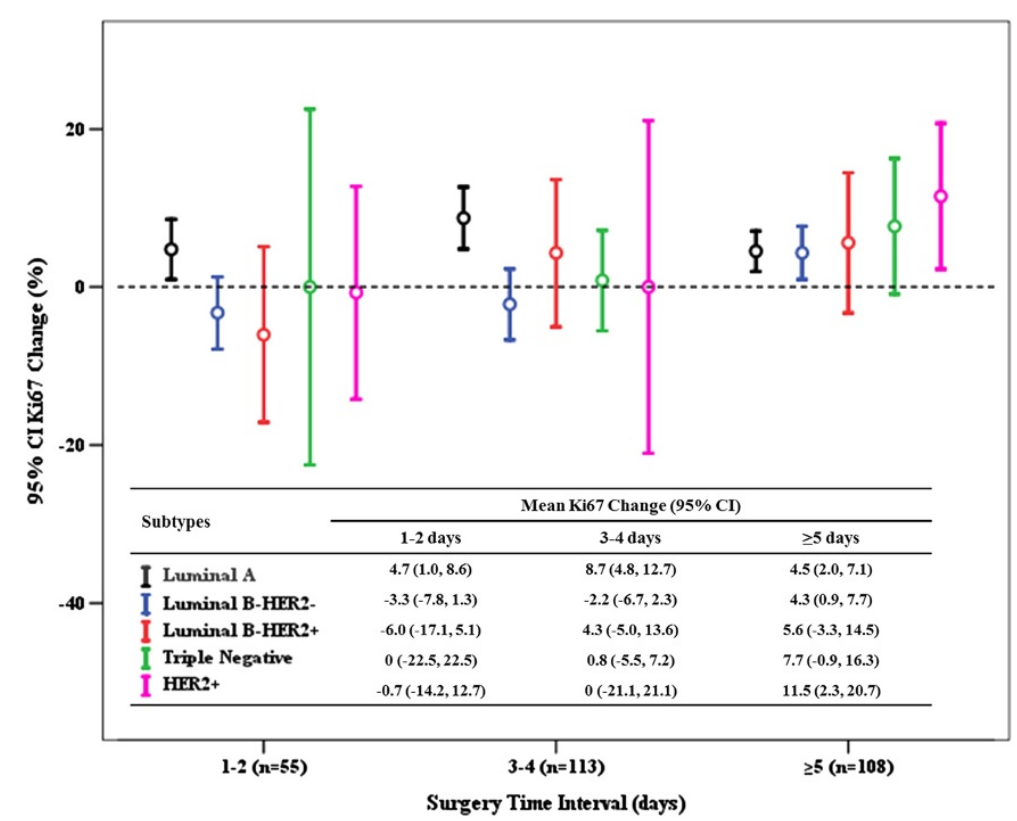

Fig. 4 Mean Ki67 change after core needle biopsy among molecular subtypes with different surgery time intervals

with longer STI had a higher Ki67 increase after CNB. TN and HER2+ breast cancer patients with longer STI had a higher degree of Ki67 increase, while this is 0 with a short STI. We propose that anti-proliferation efficacy of anti-cancer agents needs to be evaluated among different subtypes in preoperative translational trials. Patients with TN or HER2+ breast cancer probably need to be treated with a short STI or primary systemic therapy after $\mathrm{CNB}$, but this warrants further validation.

\section{Additional file}

Additional file 1: Figure S1. Mean Ki67 change after core needle biopsy among different 5 surgery time interval groups. (TIFF 39 kb)

\section{Competing interests}

The authors declare that they have no competing interests.

\section{Authors' contributions}

XSC, SJZ and KWS carried out the study conception and design. YFL, WGC and KWS participated in the study design and administrative support. XCF and XLJ carried out the histology and IHC analysis. XSC, JYW, JRH, and OH carried out the data collection and assembly. XSC, LZ, and DHG participated in data analysis, study interpretation, and drafted the manuscript. All authors read and approved the final manuscript.

\section{Acknowledgments}

This research was supported by the grants from National Natural Science Foundation of China (Grant Number: 81172520; 81202087; 81202088, 81472462); Natural Science Foundation of Shanghai Municipal Science and Technology Commission (Grant Number: 12ZR1446400); Technology Innovation Act Plan of Shanghai Municipal Science and Technology Commission (Grant Number: 14411950200, 14411950201); and Joint Research Project of the Emerging Cutting-edge Technology of Shanghai Shen-kang Hospital Development Center (Grant Number: SHDC12014103).

\section{Author details}

'Comprehensive Breast Health Center, Ruijin Hospital, Shanghai Jiaotong University School of Medicine, 197 Ruijin Er Road, Shanghai 20025, China. ${ }^{2}$ Department of Pathology, Ruijin Hospital, Shanghai Jiaotong University School of Medicine, Shanghai, China. ${ }^{3}$ University of Colorado Comprehensive Cancer Center, Aurora, CO 80045, USA.

Received: 10 November 2014 Accepted: 27 October 2015

Published online: 30 October 2015

\section{References}

1. Senkus E, Kyriakides S, Penault-Llorca F, Poortmans P, Thompson A, Zackrisson S, et al. Primary breast cancer: ESMO Clinical Practice Guidelines for diagnosis, treatment and follow-up. Ann Oncol. 2013;24 Suppl 6:vi7-23.

2. Sorlie T, Perou CM, Tibshirani R, Aas T, Geisler S, Johnsen H, et al. Gene expression patterns of breast carcinomas distinguish tumor subclasses with clinical implications. Proc Natl Acad Sci U S A. 2001;98(19):10869-74.

3. Goldhirsch A, Winer EP, Coates AS, Gelber RD, Piccart-Gebhart M, Thurlimann B, et al. Personalizing the treatment of women with early breast cancer: highlights of the St Gallen International Expert Consensus on the Primary Therapy of Early Breast Cancer 2013. Ann Oncol. 2013;24(9):2206-23.

4. Chen X, Yuan Y, Gu Z, Shen K. Accuracy of estrogen receptor, progesterone receptor, and HER2 status between core needle and open excision biopsy in breast cancer: a meta-analysis. Breast Cancer Res Treat. 2012;134(3):957-67.

5. Wolff AC, Berry D, Carey LA, Colleoni M, Dowsett M, Ellis M, et al. Research issues affecting preoperative systemic therapy for operable breast cancer. J Clin Oncol. 2008;26(5):806-13.

6. Bonanni B, Puntoni M, Cazzaniga M, Pruneri G, Serrano D, Guerrieri-Gonzaga $A$, et al. Dual effect of metformin on breast cancer proliferation in a randomized presurgical trial. J Clin Oncol. 2012;30(21):2593-600.

7. Dowsett M, Smith I, Robertson J, Robison L, Pinhel I, Johnson L, et al. Endocrine therapy, new biologicals, and new study designs for presurgical studies in breast cancer. J Natl Cancer Inst Monogr. 2011;2011(43):120-3.

8. Dowsett M, Ebbs SR, Dixon JM, Skene A, Griffith C, Boeddinghaus I, et al. Biomarker changes during neoadjuvant anastrozole, tamoxifen, or the 
combination: influence of hormonal status and HER-2 in breast cancer-a study from the IMPACT trialists. J Clin Oncol. 2005;23(11):2477-92.

9. Dowsett M, Smith IE, Ebbs SR, Dixon JM, Skene A, A'Hern R, et al. Prognostic value of Ki67 expression after short-term presurgical endocrine therapy for primary breast cancer. J Natl Cancer Inst. 2007;99(2):167-70.

10. von Minckwitz G, Schmitt WD, Loibl S, Muller BM, Blohmer JU, Sinn BV, et al. Ki67 measured after neoadjuvant chemotherapy for primary breast cancer. Clin Cancer Res. 2013;19(16):4521-31.

11. Chen $X$, Sun L, Mao Y, Zhu S, Wu J, Huang O, et al. Preoperative core needle biopsy is accurate in determining molecular subtypes in invasive breast cancer. BMC Cancer. 2013;13:390.

12. Greer LT, Rosman M, Mylander WC, Hooke J, Kovatich A, Sawyer K, et al. Does breast tumor heterogeneity necessitate further immunohistochemical staining on surgical specimens? J Am Coll Surg. 2013;216(2):239-51.

13. Gandini S, Guerrieri-Gonzaga A, Pruneri G, Serrano D, Cazzaniga M, Lazzeroni M, et al. Association of molecular subtypes with Ki-67 changes in untreated breast cancer patients undergoing pre-surgical trials. Ann Oncol. 2014;25(3):618-23

14. Paik S, Shak S, Tang G, Kim C, Baker J, Cronin M, et al. A multigene assay to predict recurrence of tamoxifen-treated, node-negative breast cancer. $\mathrm{N}$ Engl J Med. 2004;351(27):2817-26.

15. Wolff AC, Hammond ME, Schwartz JN, Hagerty KL, Allred DC, Cote RJ, et al. American Society of Clinical Oncology/College of American Pathologists guideline recommendations for human epidermal growth factor receptor 2 testing in breast cancer. J Clin Oncol. 2007;25(1):118-45.

16. Levin DC, Parker L, Schwartz GF, Rao VM. Percutaneous needle vs surgical breast biopsy: previous allegations of overuse of surgery are in error. J Am Coll Radiol. 2012:9(2):137-40.

17. Cheang MC, Chia SK, Voduc D, Gao D, Leung S, Snider J, et al. Ki67 index, HER2 status, and prognosis of patients with luminal B breast cancer. J Natl Cancer Inst. 2009:101(10):736-50.

18. Zong Y, Zhu L, Wu J, Chen X, Huang O, Fei X, et al. Progesterone Receptor Status and Ki-67 Index May Predict Early Relapse in Luminal B/HER2 Negative Breast Cancer Patients: A Retrospective Study. PLoS One. 2014;9(8):e95629.

19. Milde-Langosch K, Karn T, Muller V, Witzel I, Rody A, Schmidt M, et al. Validity of the proliferation markers Ki67, TOP2A, and RacGAP1 in molecular subgroups of breast cancer. Breast Cancer Res Treat. 2013;137(1):57-67.

20. Tagliabue E, Agresti R, Carcangiu ML, Ghirelli C, Morelli D, Campiglio M, et al. Role of HER2 in wound-induced breast carcinoma proliferation. Lancet. 2003:362(9383):527-33.

21. Carey LA, Barry WT, Pitcher B, Hoadley KA, Cheang MC, Anders CK, et al. Gene expression signatures in pre- and post-therapy $(R x)$ specimens from CALGB 40601 (Alliance), a neoadjuvant phase III trial of weekly paclitaxel and trastuzumab with or without lapatinib for HER2-positive breast cancer (BrCa). J Clin Oncol. 2014;32 suppl 15:506.

22. Gagliato DM, Gonzalez-Angulo AM, Lei X, Theriault RL, Giordano SH, Valero $\checkmark$, et al. Clinical impact of delaying initiation of adjuvant chemotherapy in patients with breast cancer. J Clin Oncol. 2014;32(8):735-44.

23. von Minckwitz G, Fontanella C. Selecting the neoadjuvant treatment by molecular subtype: how to maximize the benefit? Breast. 2013;22 Suppl 2:S149-51.

24. Wolff AC, Hammond ME, Hicks DG, Dowsett M, MCShane LM, Allison KH, et al. Recommendations for human epidermal growth factor receptor 2 testing in breast cancer: American Society of Clinical Oncology/College of American Pathologists clinical practice guideline update. J Clin Oncol. 2013;31(31):3997-4013.

\section{Submit your next manuscript to BioMed Central and take full advantage of:}

- Convenient online submission

- Thorough peer review

- No space constraints or color figure charges

- Immediate publication on acceptance

- Inclusion in PubMed, CAS, Scopus and Google Scholar

- Research which is freely available for redistribution 